released from mesenchymal stromal cells promote functional recovery and neurovascular plasticity after stroke in rats. J Cereb Blood Flow Metab 33 1711-1715.

Neuropsychopharmacology Reviews (2016) 41, 37I-372; doi: I 0.1 038/npp.2015.242

\section{New Signaling Pathway for Gut-Brain Interactions}

Inflammatory Bowel Disease (IBD), which comprises Crohn's disease and ulcerative colitis, is a chronic inflammatory condition with a relapsing course. As IBD is associated with psychiatric disorders such as depression and anxiety as well as cognitive impairment, it was suggested that these psychological factors might predispose an individual to develop the disease. Now it is clear that there is bidirectional communication between the gut and the central nervous system (Kennedy et al, 2012) and our recent finding identifies a new mechanism by which IBD might cause behavioral manifestations (Zonis et al, 2015).

The generation of new neurons continues throughout adulthood in the subgranular zone of the dentate gyrus of the hippocampus. It is well accepted that adult hippocampal neurogenesis is involved in memory and learning (Aimone et al, 2014) and various aspects of emotion and the stress response (Cameron and Glover, 2015). Thus, disruption of hippocampal neurogenesis could have profound effects on a wide range of behaviors. Among many other factors, inflammation and pro-inflammatory cytokines negatively affect neurogenesis. Peripheral inflammation can signal the brain by activating the vagus nerve and Toll-like receptors in the circumventricular organs, and pro-inflammatory cytokines can enter the brain through saturable transport systems. Engagement of this immune-to-brain communication ultimately leads to the activation of resident microglia, which is a major source of pro-inflammatory cytokines in the brain.

Previously we found that during acute systemic inflammation, cytokines upregulated in the hippocampus trigger p21 ${ }^{\text {Cip1 }}$ (p21) induction in cells of neuronal lineage (Zonis et al, 2013). p21 is a cyclin-dependent kinase inhibitor that restrains cell cycle progression, thereby reducing neurogenesis. Neuronal progenitors treated in vitro with the pro-inflammatory cytokine interleukin-6 (IL-6) exhibit p21 induction and decreased proliferation, whereas IL-6 had no effect on the proliferation of progenitor cells derived from mice lacking p21. Thus, a direct inhibitory effect of IL-6 on neurogenesis is mediated by the induction of $\mathrm{p} 21$.

Unlike acute transient inflammation, chronic inflammatory disease might have continuing and long-lasting effects on neurogenesis. To assess the effects of chronic peripheral inflammation, we utilized the dextran sodium sulfate mouse model of IBD (Strober et al, 2002). This model produces colonic epithelial cell lesions and later chronic intestinal inflammation beginning 20 days after treatment. We found increased plasma levels of IL-6, indicative of the presence of systemic inflammation and this was accompanied by increased expression of Ibal, a marker of activated microglia, and the induct-

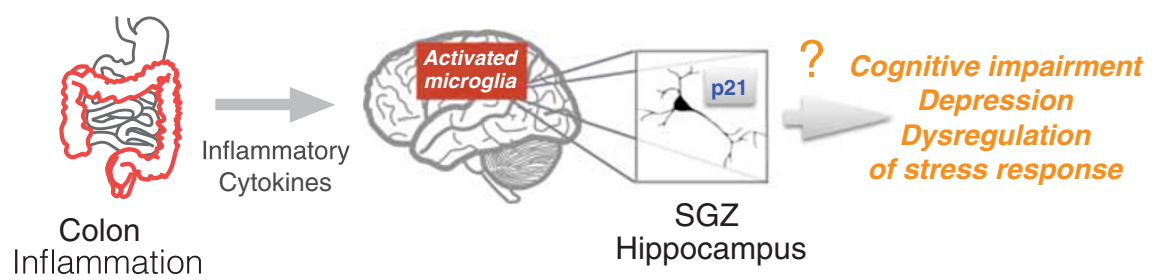

Figure 1. A proposed model for gut-hippocampus interaction. Peripheral inflammatory cytokines released during chronic intestinal inflammation activate microglia with subsequent induction of cytokines and p21 in early neuronal progenitors, effectively halting hippocampal neurogenesis and affecting behavior. SGZ-subgranular zone. ion of IL-6, IL-1 $\beta$, and p21 in the hippocampus. We also found a decrease in the number of newly developing neurons, likely due to cytokine-induced p21 expression in early neuronal progenitors. Subsequent in vitro experiments with neuronal progenitor cells confirmed that in addition to IL-6, the pro-inflammatory cytokines IL- $1 \beta$, and TNF- $\alpha$ also increase p21 expression (Zonis et al, 2015).

Our findings demonstrate that cytokine-induced p21 might have an important role in restraining neurogenesis during acute and chronic inflammation. These data reveal a previously unknown and potentially important signaling pathway for gut-brain interactions. Continuous immune signaling as a consequence of peripheral inflammation occurs in many chronic disorders, such as autoimmune disease, cancer, diabetes and obesity, and these illnesses manifest behavior abnormalities including cognitive impairment and depression. It is possible that the disruption of hippocampal neurogenesis might underlie some of the behavioral sequelae of IBD and other disorders associated with chronic inflammation (Figure 1).

\section{FUNDING AND DISCLOSURE}

This work was supported by NIH grant MH79988 and NARSAD Independent Investigator Award (VC). The authors declare no conflict of interest.

\section{ACKNOWLEDGMENTS}

The authors thank Dr Kolja Wawrowsky for help with the Figure 1.

\section{Vera Chesnokova ${ }^{1}$ and Robert N Pechnick ${ }^{2}$}

${ }^{1}$ Department of Medicine, Cedars-Sinai Medical Center, Los Angeles, CA, USA; ${ }^{2}$ Department of Basic Medical Sciences, College of Osteopathic Medicine of the Pacific, Western University of Health Sciences,

Pomona, CA, USA

E-mail: Vera.Chesnokova@cshs.org

Aimone JB, Li Y, Lee SW, Clemenson GD, Deng W, Gage FH (2014). Regulation and function of adult neurogenesis: from genes to cognition. Physiol Rev 94: 991-1026.

Cameron HA, Glover LR (2015). Adult neurogenesis: beyond learning and memory. Annu Rev Psychol 66: $53-81$. 
Kennedy PJ, Clarke G, Quigley EM, Groeger JA, Dinan TG, Cryan JF (2012). Gut memories: towards a cognitive neurobiology of irritable bowel syndrome. Neurosci Biobehav Rev 36: 310-340.

Strober W, Fuss IJ, Blumberg RS (2002). The immunology of mucosal models of inflammation. Annu Rev Immunol 20: 495-549.

Zonis S, Ljubimov VA, Mahgerefteh M, Pechnick RN, Wawrowsky K, Chesnokova V (2013). p21Cip restrains hippocampal neurogenesis and protects neuronal progenitors from apoptosis during acute systemic inflammation. Hippocampus 23 1383-1394.

Zonis S, Pechnick RN, Ljubimov VA, Mahgerefteh M, Wawrowsky K, Michelsen KS et al (2015). Chronic intestinal inflammation alters hippocampal neurogenesis. J Neuroinflammation 12: 65.

Neuropsychopharmacology Reviews (2016) 41, 372-373 doi: I 0.1038/npp.2015.237

\section{Neural Basis of Mindfulness Interventions that Moderate the Impact of Stress on the Brain}

The scientific study of mindfulness has skyrocketed. Mindfulness can be defined as 'non-judgmental attention to present-moment experiences' and is thought to comprise several complex processes, including attentional control, emotion regulation, and selfawareness (Tang et al, 2015). Although the neuroscience underlying mindfulness is at an early stage, there are some intriguing findings that begin to unravel the effects of mindfulness on mental health, stress, and resilience. For example, those individuals who rated themselves as more mindful, i.e. had greater 'dispositional mindfulness', generally report lower levels of perceived stress (Prakash et al, 2015). This is important because the level of stress is strongly related to physical and mental health as well as cortical thinning. In comparison, dispositional mindfulness has been related to structural and functional differences in several neural structures, including the medial prefrontal cortex, hippocampus, amygdala, anterior and posterior cingulate, and orbitofrontal cortex (Tang et al, 2015). Therefore, dispositional mindfulness may prove to be an important construct to examine individual differences that can help to predict risk for and relapse to mental disorders.

Mindfulness-based stress reduction (MBSR) has been proposed for almost every psychiatric condition. In a meta-analysis (Sedlmeier et al, 2012), mindfulness interventions had medium to large effect sizes for changes in emotionality and relationship issues, medium effect sizes for measures of attention, and small effect sizes for cognitive measures. MBSR has been associated with increased cortical thickness in the insula and somatosensory cortex, which can be associated with reduction of worry, state anxiety, depression, and alexithymia (Tang et al, 2015). Moreover, changes after mindfulness training in the insula have been related to increase in interoceptive awareness, i.e. the ability to monitor afferents from inside the body, which is emerging as an important construct for anxiety disorders and addiction (Paulus and Stewart, 2013). Thus, some of the same brain systems that have been implicated in dispositional mindfulness are also affected by mindfulness-based interventions and show a certain degree of plasticity of these systems.

Our understanding of the molecular mechanisms of mindfulness and changes induced by mindfulnessbased interventions is at its infancy. Recent studies have reported that MBSR training results in a smaller post-stress inflammatory response (Rosenkranz et al, 2013), which includes interleukin-6. MBSR also increased telomerase activity and those individuals with the greatest increase also reported the greatest reductions in chronic stress, anxiety, dietary restraint, dietary fat intake, cortisol, and glucose (Daubenmier et al, 2012). These findings suggest that mindfulness interventions affect both inflammatory and epigenetic mechanisms, which are important for mood and stress-related disorders, respectively. Therefore, elucidation of the molecular substrates that underlie individual differences in mindfulness may be one of the most fruitful areas for future research. Taken together, mindfulness and mindfulness-based interventions have profound effects on mental health, affect brain systems that are important for emotion regulation and self-awareness, and alter inflammatory and epigenetic responses, yet much needs to be done to make these interventions a part of precision psychiatry.

\section{FUNDING AND DISCLOSURE}

The author declares no conflict of interest.

\section{ACKNOWLEDGMENTS}

This study was funded by the William K Warren Foundation.

\section{Martin P Paulus ${ }^{1}$}

${ }^{1}$ Laureate Institute for Brain Research, Tulsa, OK, USA E-mail: mpaulus@laureateinstitute.org

Daubenmier J, Lin J, Blackburn E, Hecht FM, Kristeller J, Maninger N et al (2012). Changes in stress, eating, and metabolic factors are related to changes in telomerase activity in a randomized mindfulness intervention pilot study. Psychoneuroendocrinology 37: 917-928.

Paulus MP, Stewart JL (2013). Interoception and drug addiction. Neuropharmacology 76(Pt B): 342-350.

Prakash RS, Hussain MA, Schirda B (2015). The role of emotion regulation and cognitive control in the association between mindfulness disposition and stress. Psychol Aging 30: 160-171.

Rosenkranz MA, Davidson RJ, Maccoon DG, Sheridan JF, Kalin NH, Lutz A (2013). A comparison of mindfulness-based stress reduction and an active control in modulation of neurogenic inflammation. Brain Behav Immun 27: 174-184.

Sedlmeier P, Eberth J, Schwarz M, Zimmermann D, Haarig F, Jaeger S et al (2012). The psychological effects of meditation: a meta-analysis. Psychol Bull 138: 1139-1171.

Tang YY, Holzel BK, Posner Ml (2015). The neuroscience of mindfulness meditation. Nat Rev Neurosci 16: 213-225.

Neuropsychopharmacology Reviews (2016) 41, 373; doi: 10.1038/npp.2015.239

\section{Dynorphin, Dysphoria, and Dependence: the Stress of Addiction}

The hypothesis that the dynorphinkappa opioid receptor system may be a key component of the neuroplasticity associated with stress-induced mood disorders and the 'dark side' of 